\title{
openheart Disparity in spatial distribution of pericardial calcifications in constrictive pericarditis
}

\author{
Alpana Senapati, ${ }^{1}$ Hussain A Isma'eel, ${ }^{2}$ Arnav Kumar, ${ }^{3}$ Ayman Ayache, ${ }^{3}$ \\ Chandra K Ala, ${ }^{3}$ Dermot Phelan, ${ }^{3}$ Paul Schoenhagen, ${ }^{4}$ Douglas Johnston, ${ }^{5}$ \\ Allan L Klein ${ }^{6}$
}

To cite: Senapati $A$ Isma'eel HA, Kumar A, et al. Disparity in spatial distribution of pericardial calcifications in constrictive pericarditis. Open Heart 2018;5:e000835. doi:10.1136/ openhrt-2018-000835

\section{AS and HAl contributed equally.}

Received 13 April 2018 Revised 25 July 2018 Accepted 22 August 2018

Check for updates

(c) Author(s) (or their employer(s)) 2018. Re-use permitted under CC BY-NC. No commercial re-use. See rights and permissions. Published by BMJ.

'Department of Cardiology, Houston Methodist DeBakey Heart and Vascular Center, Houston, Texas, USA

${ }^{2}$ Division of Cardiology, Department of Internal Medicine, American University of Beirut Medical Center, Beirut, Lebanon ${ }^{3}$ Department of Cardiovascular Medicine, Cleveland Clinic , Cleveland, Ohio, USA

${ }^{4}$ Department of Diagnostic Radiology, Imaging Institute, Cleveland Clinic , Cleveland, Ohio, USA

${ }^{5}$ Department of Thoracic and Cardiovascular Surgery, Heart and Vascular Institute, Cleveland Clinic, Cleveland, Ohio, USA

${ }^{6}$ Center for the Diagnosis and Treatment of Pericardial Diseases, Cleveland Clinic , Heart and Vascular Institute, Cleveland, Ohio, USA

Correspondence to Dr Allan L Klein; kleina@ccf.org

\section{ABSTRACT}

Background Pericardial calcification is seen among patients with constrictive pericarditis (CP). However, the pattern of pericardial calcium distribution and the association with clinical outcomes and imaging data are not well described.

Methods This was a retrospective study from 2007 to 2013 to evaluate the pattern of pericardial calcium distribution by $\mathrm{CT}$ in $\mathrm{CP}$ using a semiquantitative calcium scoring system to calculate total pericardial calcium burden and distribution. Calcium localisation was allocated to 20 regions named after the corresponding heart structure. Baseline clinical data, imaging data and clinical outcomes were collected and compared between the calcified pericardium and non-calcified pericardium groups. We assessed the effect of pericardial calcium on clinical outcomes and echocardiographic data between the two groups.

Results Of the 123 consecutive patients with CP (93 male; mean age $61 \pm 13$ years) between 2007 and 2013, 49 had calcified pericardium and 74 had non-calcified pericardium. Distribution of calcium on the left ventricle (LV) basal anterior, mid-anterior and apical segments in addition to right ventricle (RV) apical segment was involved in $<30 \%$ of the cases with the remaining segments involved in $>35 \%$ of cases. A potential protective role of RV calcium on regional myocardial mechanics was noted. Conclusion Preferential distribution of calcium in CP in a partial band-like pattern (from basal anterolateral LV going inferiorly and then encircling the heart to reach the RV outflow tract) with extension into the mitral and tricuspid annuli was noted. Pericardial calcium was not significantly associated to clinical outcomes.

\section{INTRODUCTION}

Constrictive pericarditis (CP) is a debilitating disease caused by a thickened and fibrosed pericardium limiting diastolic filling of the ventricles. It usually results from chronic inflammation due to an initial episode of acute pericarditis as an inciting event. Most common aetiologies in the USA and Europe are a result of idiopathic/viral or postpericardiotomy syndrome whereas in low/middle-income countries, tuberculosis is a prevalent

\section{Key questions}

What is already known about this subject?

- Constrictive pericarditis is a debilitating disease leading to significant morbidity and mortality. CT provides excellent anatomic assessment of the pericardium prior to surgical planning for pericardiectomy.

- However, the pattern of pericardial calcium distribution and the association with clinical outcomes are not well described.

What does this study add?

- Pericardial calcification was found to be located in a circumferential band-like pattern sparing the basal anterior left ventricle and apical regions of the left and right ventricles, with extension into the mitral and tricuspid annuli in more than $40 \%$ of the patients.

- In this study, a potential protective role of pericardial calcium around the right ventricle was noted, with lower grades of tricuspid regurgitation.

How might this impact on clinical practice?

- Evaluating the distribution of pericardial calcification may aid better understanding of the effect of calcium on regional myocardial mechanics and function.

- Further studies with a quantitative assessment of pericardial calcification and effect on clinical outcomes are needed.

cause. ${ }^{1}$ Multimodality imaging with echocardiogram (ECHO), cardiac MRI (CMR) and CT is useful to guide the management and diagnosis of CP. The diagnosis of CP can be challenging, therefore algorithms are available to guide the diagnostic approach and need for additional imaging. ${ }^{2}$ CT provides excellent anatomic assessment of the pericardium and is the preferred imaging modality to assess pericardial calcium (PC) prior to surgical planning. ${ }^{3}$ Pericardial calcification occurs frequently in CP; however, calcification is not always associated with constriction. ${ }^{4}$ The impact of pericardial calcification on survival after pericardiectomy has been controversial. Studies have shown pericardial calcification 
to be an independent predictor of negative perioperative outcomes but no impact on long-term survival. ${ }^{5}$ However, other studies have shown a negative impact on long-term survival $^{6}$ highlighting the discrepancy in the consensus on pericardial calcification and long-term survival. The pattern of calcium distribution in CP is not well described and the association between severity of calcification and clinical outcomes has not been evaluated. A PC scoring system has not been established to evaluate the effects of calcium distribution on ECHO variables, surgical, or clinical outcomes in CP.

\section{METHODS \\ Patient population}

This is a retrospective study of patients referred to our centre between 2007 and 2013 with CP who had CT with or without contrast imaging. The diagnosis of CP was confirmed through evidence of constriction on one or more of the following: (A) ECHO, (B) CMR or (C) cardiac catheterisation along with clinical characteristics such as symptoms of right heart failure. Echocardiographic features of constriction included: septal bounce, interventricular dependence, respiratory variation of early mitral inflow velocity $>25 \%$ and tricuspid early inflow velocity $>40 \%$, and inferior vena cava (IVC) plethora. Features of constriction on CMR included: pericardial thickening, conical deformity of the ventricles and interventricular dependence. Cardiac catheterisation features of constriction include: elevated right atrial pressures, elevated right ventricle (RV) and left ventricle (LV) end-diastolic pressures with dip and plateau sign, and interventricular dependence. ${ }^{1}$

Baseline clinical characteristics, CP aetiology, presurgical New York Heart Association (NYHA) class, medical comorbidities, medications, laboratory values and clinical outcomes were collected and compared between the calcified pericardium and non-calcified pericardium groups. Clinical and imaging data were collected through review of electronic medical record system. Aetiology was divided into idiopathic, infectious, radiation, postpericardiotomy syndrome, trauma, autoimmune, neoplastic or uraemic. The aetiologies were defined based on current definitions and guidelines. ${ }^{17}$

\section{Clinical outcomes}

Clinical outcomes of all-cause mortality, need for pericardiectomy and surgical outcomes were assessed. All-cause mortality was identified either through use of social security index data or the electronic medical record system. Surgical outcomes were collected at index hospitalisation for all patients who underwent pericardiectomy. Concomitant procedure was defined as coronary artery bypass, valve repair and/or replacement. In-hospital mortality was defined as any patient who died during the initial index hospitalisation. Operative mortality was defined as any patient who died during the index surgery within a 30-day postprocedure period. Readmission within 30 days

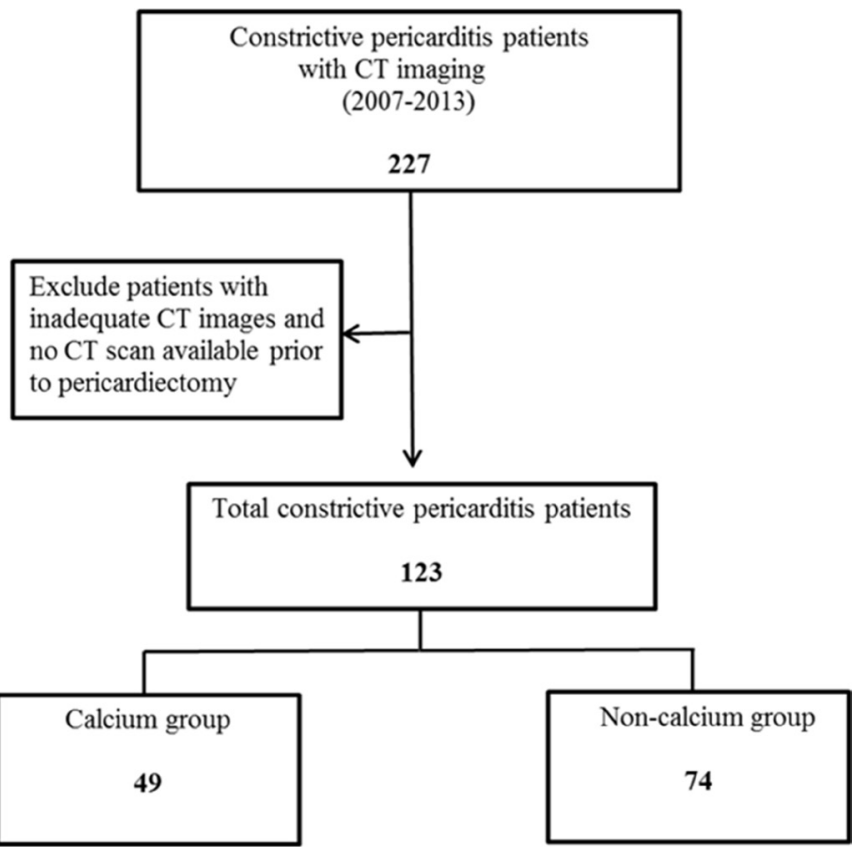

Figure 1 Constrictive pericarditis patient population. The chart consists of the overall constrictive pericarditis patient population divided into segments excluding patients who did not meet inclusion criteria. This was divided into our total constrictive pericarditis population and further divided into calcified pericardium and non-calcified pericardium groups.

was reviewed through the use of the electronic medical record system and defined as any admission that required a hospital admission, excluding office visits and emergency department visits.

\section{Calcium scoring}

We developed a semiquantitative PC scoring system as a measure of pericardial calcification burden to evaluate the distribution of calcium in patients with calcified pericardium. All patients were scanned on standard multidetector CT scanner. PC was identified visually as pericardial segments with high Hounsfield unit. The scoring system allocated calcium into 20 regions named after the corresponding heart structure: the LV and RV were further divided in short axis into four basal, four mid and three apical divisions for the LV and four basal, four mid (both in short axis) divisions and one apical-lateral division (from apical four-chamber view) for the RV (figures 1 and 2). In addition, calcifications in the pericardium surrounding the left and right atria, or extending into the mitral or tricuspid annuli were also noted. PC scores as a measure of pericardial calcification burden were calculated with 1 point given for each involved calcified area. Total PC scores (LV and RV segments only) and regional PC scores (LV, RV, annuli and atria) were then calculated and graphed for each area. Only acceptable CT scans prior to surgery were used for analysis based on the quality of scan to detect calcium in all visualised segments. Patients were excluded if CT scans were not available prior to surgery. 


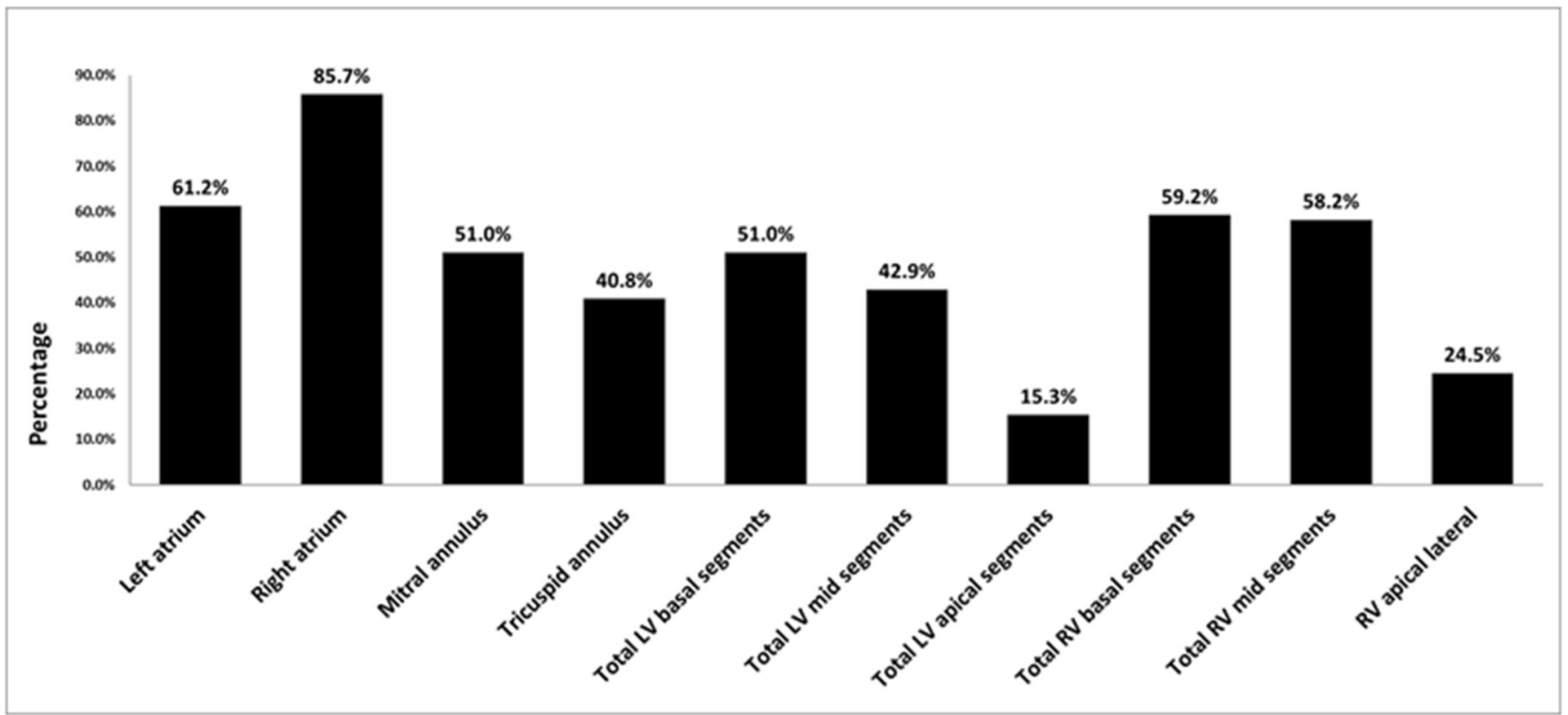

Figure 2 Spatial distribution of pericardial calcifications. This figure illustrates the frequency of pericardial calcium location corresponding to the various heart structures: left and right atria, mitral and tricuspid annuli, total left ventricle (LV) basal, mid and apical segments, and the total right ventricle (RV) basal, mid and apical segments.

\section{Statistical methods}

Statistical analysis was performed using IBM SPSS Statistics V.20.0 (IBM). All continuous variables are expressed as mean $\pm \mathrm{SD}$ and categorical variables as frequency with percentages. $\mathrm{P}$ values were obtained for all reported variables and significance defined as $p \leq 0.05$. The Shapiro-Wilk test was used to test for normality of continuous variables, with a $p$ value $\leq 0.05$ signifying non-normal data. Continuous variables were also tested for homogeneity of variances using Levene's test for normally distributed data, and analysis of variance for non-normal data, with a $p$ value $\leq 0.05$ signifying unequal variances. For continuous variables, significance was determined using the independent samples t-test, Mann-Whitney U test, or the Mood's median test, depending on normality and variance of the data. The t-test was used on normally distributed data with equal variance and the Welch's t-test statistic was used for normally distributed data with unequal variance. The Mann-Whitney $U$ test was used on non-normal data with equal variance, and the Mood's median test was used on non-normal data with unequal variance. Univariable logistic regression and linear regression models were used to assess predictors of all-cause mortality and total and regional calcium scores on clinical outcomes and echocardiographic variables for patients with PC. Logistic and ordinal (for ranked data) regression models are reported with OR, 95\% CIs and $\mathrm{p}$ value. Linear regression is reported with correlation coefficient $R^{2}$, beta $(\beta)$ and $p$ value. Pearson's correlation was used to determine the relationship between LV ejection fraction (LVEF) and total calcium. Correlations are reported with the correlation coefficient $\mathrm{R}^{2}$ and corresponding $\mathrm{p}$ value.

\section{RESULTS}

\section{Patient characteristics}

A total of 123 consecutive patients with CP and CT scans available for review were identified through the Cleveland Clinic pericardial database from 2007 to 2013. Patients were classified into calcified pericardium $(n=49)$ and non-calcified pericardium $(\mathrm{n}=74)$ groups (figure 3 ). Baseline clinical characteristics, CP aetiology and clinical outcomes were collected as illustrated in table 1. Mean age was $61 \pm 13$ years $(76 \%$ male) in overall population. Idiopathic aetiology $(48 \%)$ was most common and there was no significant difference in aetiologies between the two groups. The use of diuretics, presence of atrial fibrillation and chronic obstructive pulmonary disease (COPD) were significantly higher in the calcified pericardium group. There was no significant difference in age, gender, NYHA class, pericardiectomy or mortality in the two groups.

\section{Echocardiographic variables}

Routine ECHO variables were measured between the calcium and non-calcium groups and outlined in table 2. Left atrial diameter was larger in calcified group 5.21 \pm 0.97 $\mathrm{cm}$ vs $4.10 \pm 0.95 \mathrm{~cm}$ in the non-calcified group $(\mathrm{p}=0.002)$; however, left atrial volume index was not statistically significant $(\mathrm{p}=0.077)$. There was significantly higher incidence of diastolic septal bounce and larger IVC diameters in the calcified pericardial group versus the non-calcified group ( $\mathrm{p}=0.004, \mathrm{p}=0.001)$. Mitral medial $\mathrm{e}^{\prime}$ was also higher in the calcified group $(\mathrm{p}=0.006)$. There were no other significant differences in echocardiographic parameters between the two groups. 


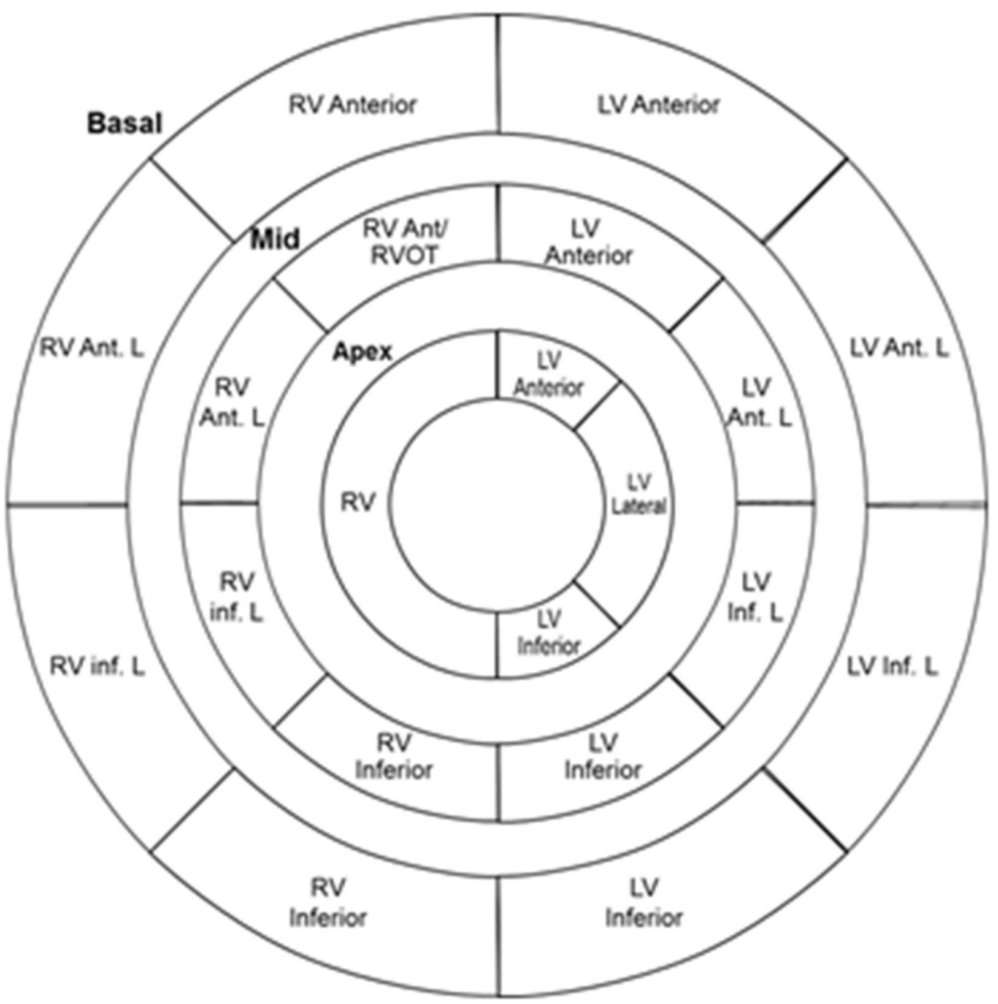

Figure 3 Left ventricle (LV) and right ventricle (RV) spatial distribution of pericardial calcifications. This figure illustrates the allocation of pericardial calcium localisation to the 20 areas (LV and RV only) for our semiquantitative scoring system called after the corresponding heart structure: the LV and RV were further divided in short axis into four basal, four mid and three apical divisions for the LV and four basal, four mid (both in short axis) divisions and one apical-lateral division (from apical fourchamber view) for the RV. Segments were then colour coded to illustrate the degree of calcium found in these areas labelled P for pericardium. Ant, anterior; Inf, inferior; L, lateral; RVOT, right ventricle outflow tract. 
Table 1 Clinical characteristics

\begin{tabular}{|c|c|c|c|c|}
\hline & $\begin{array}{l}\text { Overall } \\
(n=123)\end{array}$ & $\begin{array}{l}\text { Calcified } \\
(n=49)\end{array}$ & $\begin{array}{l}\text { Non-calcified } \\
(n=74)\end{array}$ & $P$ values \\
\hline Age & $61 \pm 13$ & $63 \pm 13$ & $59 \pm 14$ & 0.073 \\
\hline Male & $93(76)$ & $39(80)$ & $54(73)$ & 0.403 \\
\hline All-cause mortality & $26(21)$ & $13(27)$ & $13(18)$ & 0.223 \\
\hline \multicolumn{5}{|l|}{ NYHA } \\
\hline 1 & $13(11)$ & $3(6)$ & $10(14)$ & 0.192 \\
\hline$\|$ & $57(46)$ & $22(45)$ & $35(47)$ & 0.794 \\
\hline III & $50(41)$ & $21(43)$ & $29(39)$ & 0.685 \\
\hline IV & $3(2)$ & $3(6)$ & $0(0)$ & 0.061 \\
\hline Ejection fraction & $57 \pm 8.1$ & $56.1 \pm 8.2$ & $57.5 \pm 8.1$ & 0.358 \\
\hline \multicolumn{5}{|l|}{ CP aetiology } \\
\hline Idiopathic & $59(48)$ & $28(57)$ & $31(42)$ & 0.097 \\
\hline Infectious & $4(3)$ & $0(0)$ & $4(5)$ & 0.098 \\
\hline Radiation & $15(12)$ & $4(8)$ & $11(15)$ & 0.266 \\
\hline Postpericardiotomy syndrome & $31(25)$ & $14(29)$ & $17(23)$ & 0.484 \\
\hline Trauma & $3(2)$ & $1(2)$ & $2(3)$ & 1 \\
\hline Autoimmune & $6(5)$ & $1(2)$ & $5(7)$ & 0.401 \\
\hline Neoplastic & $4(3)$ & $0(0)$ & $4(5)$ & 0.150 \\
\hline Uraemic & $1(0.8)$ & $1(2)$ & $0(0)$ & 0.398 \\
\hline Pericardiectomy & $64(52)$ & $30(61)$ & $34(46)$ & 0.097 \\
\hline \multicolumn{5}{|l|}{ Comorbidities } \\
\hline Hypertension & $71(58)$ & $25(51)$ & $46(62)$ & 0.221 \\
\hline Hyperlipidaemia & $55(45)$ & $20(41)$ & $35(47)$ & 0.479 \\
\hline Diabetes mellitus & $33(27)$ & $11(23)$ & $22(30)$ & 0.372 \\
\hline CAD & $55(44)$ & $19(39)$ & $35(47)$ & 0.351 \\
\hline CHF & $59(48)$ & $27(55)$ & $32(43)$ & 0.197 \\
\hline CKD & $27(22)$ & $09(18)$ & $18(24)$ & 0.435 \\
\hline Atrial fibrillation & $56(46)$ & $32(65)$ & $24(32)$ & $<0.001$ \\
\hline COPD & $23(19)$ & $14(29)$ & $09(12)$ & 0.022 \\
\hline Smoking history & $72(59)$ & $33(67)$ & $39(53)$ & 0.107 \\
\hline \multicolumn{5}{|l|}{ Medication use } \\
\hline Aspirin & $46(37 \%)$ & $16(33 \%)$ & $30(41 \%)$ & 0.376 \\
\hline Colchicine & $16(13 \%)$ & $4(8 \%)$ & $12(16 \%)$ & 0.194 \\
\hline NSAID & $20(16 \%)$ & $6(12 \%)$ & $14(19 \%)$ & 0.326 \\
\hline Steroid & $24(20 \%)$ & $6(12 \%)$ & $18(24 \%)$ & 0.098 \\
\hline DMARD & $2(2 \%)$ & 0 & $2(3 \%)$ & 0.517 \\
\hline Diuretic & $81(66 \%)$ & $39(80 \%)$ & $42(57 \%)$ & 0.009 \\
\hline \multicolumn{5}{|l|}{ Lab findings* } \\
\hline White cell count & $8 \pm 3.4$ & $7.6 \pm 3.8$ & $8.2 \pm 3.1$ & 0.331 \\
\hline Haemoglobin & $12.2 \pm 2.1$ & $12.7 \pm 2.1$ & $11.8 \pm 2.1$ & 0.019 \\
\hline Platelets & $226 \pm 105$ & $195 \pm 82$ & $246 \pm 114$ & 0.005 \\
\hline Sodium & $137.8 \pm 3.9$ & $138.4 \pm 4.5$ & $137.5 \pm 3.5$ & 0.246 \\
\hline Potassium & $4.1 \pm 0.6$ & $4.0 \pm 0.6$ & $4.1 \pm 0.6$ & 0.388 \\
\hline Creatinine & $1.3 \pm 1.7$ & $1.2 \pm 0.8$ & $1.4 \pm 2.1$ & 0.554 \\
\hline Albumin & $3.8 \pm 0.6$ & $4 \pm 0.6$ & $3.6 \pm 0.6$ & 0.002 \\
\hline
\end{tabular}

Data were summarised as frequency $\mathrm{n}(\%)$ for descriptive data and mean \pm SD for continuous data.

${ }^{*}$ Total patients included $n=120: 47$ in calcified pericardium group and 73 in non-calcified pericardium group.

$\mathrm{CAD}$, coronary artery disease; $\mathrm{CHF}$, congestive heart failure; CKD, chronic kidney disease; COPD, chronic obstructive pulmonary disease; $\mathrm{CP}$, constrictive pericarditis; DMARD, disease-modifying antirheumatic drug; NSAID, non-steroidal anti-inflammatory drug; NYHA, New York Heart Association. 
Table 2 Echocardiogram data

\begin{tabular}{|c|c|c|c|c|}
\hline & $\begin{array}{l}\text { Overall } \\
(n=123)\end{array}$ & $\begin{array}{l}\text { Calcified } \\
(n=49)\end{array}$ & $\begin{array}{l}\text { Non-calcified } \\
(n=74)\end{array}$ & $P$ values \\
\hline LV end-diastolic dimension, $\mathrm{cm}(\mathrm{n}=84,27,57)$ & $4.16 \pm 0.76$ & $4.17 \pm 0.67$ & $4.15 \pm 0.80$ & 0.937 \\
\hline LV end-systolic dimension, $\mathrm{cm}(\mathrm{n}=81,25,56)$ & $2.80(2.30-3.20)$ & $3.00(2.40-3.30)$ & $2.70(2.30-3.13)$ & 0.188 \\
\hline LV septal thickness, $\mathrm{cm}^{*}$ & $1.00(0.80-1.20)$ & $1.00(0.80-1.18)$ & $1.00(0.80-1.20)$ & 0.591 \\
\hline LV posterior wall thickness, $\mathrm{cm}^{*}$ & $1.00(0.90-1.10)$ & $1.00(0.90-1.08)$ & $1.00(0.90-1.13)$ & 0.724 \\
\hline LA diameter, $\mathrm{cm}(\mathrm{n}=36,15,21)$ & $4.56 \pm 1.10$ & $5.21 \pm 0.97$ & $4.10 \pm 0.95$ & 0.002 \\
\hline LA volume index, $\mathrm{mL} / \mathrm{m}^{2}(\mathrm{n}=59,26,33)$ & $29.40(22.40-39.00)$ & $31.90(23.50-42.88)$ & $27.30(18.50-33.50)$ & 0.077 \\
\hline LV EDV, mL† & $84.00(67.00-105.50)$ & $75.00(64.00-89.50)$ & $87.50(68.50-107.50)$ & 0.215 \\
\hline LV ESV, mL† & $32.00(23.00-44.50)$ & $31.00(22.50-40.50)$ & $35.00(24.50-44.50)$ & 0.565 \\
\hline $\mathrm{EF}, \%$ & $56.98 \pm 8.14$ & $56.14 \pm 8.22$ & $57.53 \pm 8.10$ & 0.358 \\
\hline MV E, cm/s $(n=99,38,61)$ & $90.00(75.00-110.75)$ & $86.75(77.61-112.88)$ & $90.00(73.00-108.50)$ & 0.599 \\
\hline$\%$ MV E respiratory change $(n=90,36,54)$ & $14.43(5.76-19.79)$ & $16.40(5.27-18.99)$ & $12.96(6.87-21.41)$ & 0.814 \\
\hline MV A, cm/sł & $53.24 \pm 17.45$ & $48.47 \pm 17.46$ & $56.17 \pm 16.99$ & 0.071 \\
\hline MV E/A ratioł & $1.73(1.35-2.18)$ & $1.82(1.51-2.50)$ & $1.62(1.30-2.13)$ & 0.133 \\
\hline Deceleration time, ms§ & $148.00(126.75-174.38)$ & $140.20(121.13-164.50)$ & $154.00(138.63-179.13)$ & 0.091 \\
\hline TV E, cm/sף & $61.75(52.13-69.00)$ & $64.75(52.75-71.00)$ & $60.50(51.75-66.00)$ & 0.232 \\
\hline TV e' lateral, cm/s $(\mathrm{n}=48,16,32)$ & $10.12 \pm 3.64$ & $11.30 \pm 3.28$ & $9.53 \pm 3.72$ & 0.083 \\
\hline \% TV E respiratory changeף & $-35.28 \pm 17.41$ & $-34.70 \pm 15.09$ & $-35.76 \pm 19.33$ & 0.713 \\
\hline Septal bounce $(n=91,36,55)$ & $91(82)$ & $36(97)$ & $55(73)$ & 0.004 \\
\hline IVC, $\mathrm{cm}(\mathrm{n}=84,34,50)$ & $2.60(2.28-2.83)$ & $2.80(2.43-3.00)$ & $2.50(2.13-2.70)$ & 0.001 \\
\hline MV e' medial, cm/s§ & $9.00(8.00-12.00)$ & $10.50(9.00-14.00)$ & $9.00(8.00-11.00)$ & 0.006 \\
\hline MV $\mathrm{e}^{\prime}$ lateral, $\mathrm{cm} / \mathrm{s}^{\star \star}$ & $10.00(8.00-12.25)$ & $11.00(8.00-14.00)$ & $10.00(8.00-12.00)$ & 0.068 \\
\hline Average $E / e^{\prime} M V(n=87,34,53)$ & $8.65(6.22-11.81)$ & $8.07(5.97-11.23)$ & $8.76(6.88-13.33)$ & 0.130 \\
\hline Annulus reversus ${ }^{\star *}$ & $84(42)$ & 33 (51.52) & $51(35.29)$ & 0.176 \\
\hline
\end{tabular}

Data were summarised as frequency $\mathrm{n}(\%)$ for descriptive data and mean \pm SD for continuous data.

Total patients in each group where $n=$ total, calcified, non-calcified group: * $(n=82,26,56), \dagger(n=55,19,36), \ddagger(n=71,27,44), \S(88,34,54)$, १(62,28,34), **(84,33,51).

EDV, end-diastolic volume; EF, ejection fraction; ESV, end-systolic volume; IVC, inferior vena cava; LA, left atrium; LV, left ventricle; MV, mitral valve; TV, tricuspid valve.

\section{Surgical data}

Surgical outcomes were collected at index hospitalisation for all patients who underwent pericardiectomy as outlined in table 3 . Of the 64 patients, there were $2(3 \%)$ in hospital mortality and $1(2 \%)$ operative mortality. Five patients $(8 \%)$ did not have follow-up at 30 days, but had phone follow-up $<30$ days. There was no significant difference in the surgical outcomes between the two groups.

\section{Calcium scoring}

PC spatial distribution for the calcified pericardium group is illustrated in figure 1. Figure 2 further illustrates pericardial spatial distribution within the LV and RV basal, mid and apical regions. Calcium involvement of the LV basal anterior, mid-anterior and apical segments in addition to RV apical segment was involved in $<30 \%$ of the cases. The remaining segments were involved in $>35 \%$ of cases. Of note, $51 \%$ and $41 \%$ had PC extending into the mitral and tricuspid annuli. Two of the 49 patients in the calcified pericardium group had calcium localised to the atria or LV apical cap.

Tables 4 and 5 illustrate the univariable logistic regression and linear regression models used to assess predictors of all-cause mortality and effect of different variables on total PC scores. Total PC scores and regional PC scores (LV, RV and annuli) were used to assess predictors of clinical outcomes and echocardiographic variables. The multinomial logistic regression model showed that albumin and NYHA play a significant role in predicting all-cause mortality with higher albumin scores associated with lower mortality and higher NYHA associated with higher mortality. The linear regression model showed that with higher RV scores, \% respiratory change in tricuspid valve decreased $\left(R^{2}=0.149\right.$, $\beta=-2.072, p=0.043)$ and tricuspid regurgitation grade also decreased $(\mathrm{OR}=0.743, \mathrm{p}=0.008)$. There was no significant correlation between LVEF and total calcium $\left(\mathrm{R}^{2}=-0.044, \mathrm{p}=0.764\right)$. 


\begin{tabular}{|c|c|c|c|c|}
\hline & $\begin{array}{l}\text { Overall } \\
(n=64)\end{array}$ & $\begin{array}{l}\text { Calcified } \\
(n=30)\end{array}$ & $\begin{array}{l}\text { Non-calcified } \\
(n=34)\end{array}$ & $P$ values \\
\hline \multicolumn{5}{|l|}{ Surgery approach } \\
\hline Median sternotomy & $53(83 \%)$ & $26(87 \%)$ & $27(79 \%)$ & 0.443 \\
\hline Anterior thoracotomy & $11(17 \%)$ & $4(13 \%)$ & $7(21 \%)$ & 0.443 \\
\hline Redo sternotomy & $18(28 \%)$ & $6(20 \%)$ & $12(35 \%)$ & 0.174 \\
\hline Use of CPB & $46(72 \%)$ & $22(73 \%)$ & $24(71 \%)$ & 0.807 \\
\hline Concomitant procedure & $26(41 \%)$ & $10(33 \%)$ & $16(47 \%)$ & 0.265 \\
\hline \multicolumn{5}{|l|}{ Surgical outcomes } \\
\hline LOS $>5$ days & $60(94 \%)$ & $30(100 \%)$ & $30(88 \%)$ & 0.116 \\
\hline In-hospital mortality & $2(3 \%)$ & $1(3 \%)$ & $1(3 \%)$ & 1 \\
\hline Operative mortality & $1(2 \%)$ & $1(3 \%)$ & 0 & 0.47 \\
\hline ICU stay $>8$ days & $8(13 \%)$ & $5(17 \%)$ & $3(9 \%)$ & 0.458 \\
\hline Readmission within 30 days & $8(13 \%)$ & $5(17 \%)$ & $3(9 \%)$ & 0.458 \\
\hline Reoperation for bleeding & $3(5 \%)$ & $2(7 \%)$ & $1(3 \%)$ & 0.596 \\
\hline
\end{tabular}

Data were summarised as frequency $\mathrm{n}(\%)$ for descriptive data and mean \pm SD for continuous data.

$\mathrm{CPB}$, cardiopulmonary bypass;ICU, intensive care unit;LOS, length of stay (in days).

\section{DISCUSSION}

The results of our study show the preferential distribution of calcium in CP in a partial band-like pattern (from basal anterolateral LV going inferiorly and then encircling the heart to reach the RV outflow tract) with extension into the mitral and tricuspid annuli. Calcium involvement of the pericardial segments corresponding to the LV basal anterior, mid-anterior and apical segments in addition to RV apical segment was involved in $<30 \%$ of the cases. The remaining segments were involved in $>35 \%$ of cases. Once pericardial calcifications are present, $>40 \%$ of patients will have pericardial calcifications extending into either the mitral or tricuspid annuli. Several studies have investigated perioperative complications and outcomes after pericardiectomy. ${ }^{78}$ In our cohort, there was no significant difference in surgical outcomes between the calcified and non-calcified pericardium groups.

As in previous studies, idiopathic CP was the most common aetiology in our study. ${ }^{9}$ Higher diuretic use, incidence of atrial fibrillation, COPD, larger atria and IVC diameters were also found in the calcified pericardium group, suggesting a more chronic and symptomatic

\begin{tabular}{|c|c|c|c|c|}
\hline Independent variables & Dependent variables & OR & $95 \% \mathrm{Cl}$ & $P$ values \\
\hline \multirow[t]{3}{*}{ Total PC score } & Death & 0.935 & (0.807 to 1.084$)$ & 0.373 \\
\hline & NYHA & 0.959 & (0.851 to 1.082$)$ & 0.498 \\
\hline & Pericardiectomy & 1.033 & (0.907 to 1.176$)$ & 0.624 \\
\hline NYHA & Death & 3.946 & (1.468 to 10.608$)$ & 0.007 \\
\hline Ejection fraction & & 0.960 & (0.904 to 1.020$)$ & 0.188 \\
\hline Age & & 1.028 & (0.981 to 1.077$)$ & 0.242 \\
\hline Albumin & & 0.305 & (0.127 to 0.735$)$ & 0.008 \\
\hline Sodium & & 1.003 & (0.881 to 1.142$)$ & 0.963 \\
\hline \multirow[t]{2}{*}{ Total LV scores } & MR grade & 0.891 & (0.736 to 1.079$)$ & 0.236 \\
\hline & Annulus reversus & 1.258 & (0.979 to 1.617$)$ & 0.073 \\
\hline Total RV scores & TR grade & 0.743 & (0.597 to 0.926$)$ & 0.008 \\
\hline \multirow[t]{2}{*}{ Mitral annulus } & MR grade & 0.868 & (0.261 to 2.888$)$ & 0.817 \\
\hline & Annulus reversus & 3.086 & (0.734 to 12.981$)$ & 0.124 \\
\hline Tricuspid annulus & TR grade & 2.568 & (0.736 to 8.965$)$ & 0.139 \\
\hline
\end{tabular}

LV, left ventricle; MR, mitral regurgitation; NYHA, New York Heart Association; PC, pericardial calcium; RV, right ventricle; TR, tricuspid regurgitation. 


\begin{tabular}{|c|c|c|c|c|}
\hline Independent variables & Dependent variables & $\mathbf{R}^{2}$ & $\beta$ & $P$ values \\
\hline \multirow[t]{7}{*}{ Total LV scores } & MV E & 0.047 & -1.832 & 0.190 \\
\hline & $\%$ respiratory MV E & 0.005 & 0.230 & 0.675 \\
\hline & MV E/A ratio & 0.001 & -0.014 & 0.895 \\
\hline & DT & 0.017 & 1.711 & 0.461 \\
\hline & MV e' medial & 0.034 & 0.253 & 0.294 \\
\hline & MV e' lateral & 0.035 & -0.254 & 0.300 \\
\hline & Average E/e' MV & 0.004 & -0.076 & 0.731 \\
\hline \multirow[t]{3}{*}{ Total RV scores } & TVE & 0.054 & -2.246 & 0.234 \\
\hline & TV e' lateral & 0.048 & 0.320 & 0.415 \\
\hline & \% respiratory TV E & 0.149 & -2.072 & 0.043 \\
\hline Age & Total PC score & 0.012 & -0.037 & 0.463 \\
\hline Gender & & 0.012 & 1.236 & 0.445 \\
\hline Smoking & & 0.006 & 0.737 & 0.597 \\
\hline Creatinine & & 0.062 & -1.337 & 0.092 \\
\hline Albumin & & 0.004 & -0.464 & 0.680 \\
\hline Body mass index & & 0.062 & 0.176 & 0.210 \\
\hline
\end{tabular}

$\%$ resp, percent respiratory variation; A, peak late mitral diastolic flow velocity; DT, deceleration time; E, peak early diastolic flow velocity; e', early mitral annular tissue diastolic velocity; LV, left ventricle; MV, mitral valve; PC, pericardial calcium; $\mathrm{R}^{2}$, correlation coefficient; RV, right ventricle; TV, tricuspid valve.

patient population. The distribution of pericardial calcifications has been described in other case reports and small studies, but has not been evaluated in a similar sized case series. ${ }^{11-15}$ The relative sparing of the pericardial segments corresponding to the basal anterior LV and apical segments with significant involvements of the other segments brings out two questions ${ }^{1}$ : what is the mechanism favouring this pattern of calcium deposition, and ${ }^{2}$ how does this impact the mechanics of the heart. However, we believe that pericardial calcification can be viewed as the end product of chronic inflammation. In our study, aspirin use was associated with lower total PC scores, suggesting perhaps the important role for anti-inflammatory therapy in this process. Thus, the calcified parts are the most affected and if the innervation of the heart plays a role in the aetiology of the insult then this calcification pattern follows the heart innervation pattern. Alternatively, we cannot exclude a simple dependent mechanism of inflammatory material that follows the patients being in a supine position. This, however, will not explain the RV outflow tract involvement. Studies have shown that patients with CP have lower lateral annulus tissue Doppler compared with the medial annulus, referred to as 'annulus reversus'. ${ }^{16-18}$ This finding parallels the increased calcium involvement of the pericardial segments corresponding to the lateral wall often extending into the mitral annulus, thereby limiting lateral annular velocity due to tethering from fibrotic or calcified pericardium. Further, higher RV calcium scores were shown to have lower grades of tricuspid regurgitation and lower respiratory variation, suggesting perhaps a protective role of RV calcium with regional mechanics.

Pericardial calcification's impact on survival has been controversial with some studies showing a negative impact on survival and others showing no impact. ${ }^{569}$ In our study, clinical outcomes of mortality and pericardiectomy between the two groups were not found to be statistically significant and total PC score did not significantly predict clinical outcomes. Previous studies have shown poor surgical outcomes with advanced NYHA, radiation aetiology, chronic lung disease with renal failure, use of cardiopulmonary bypass and previous cardiac surgery to be predictors of worse outcomes. ${ }^{78}$ In our study, the presence of calcium did not significantly predict adverse surgical outcomes or all-cause mortality. The number included in the cohort may explain the absence of statistical association.

\section{Study limitations}

This is a retrospective study at a single institution with a relatively small sample size, therefore limiting power and introducing potential selection bias. The retrospective design, lack of a true quantitative method to assess PC and limited follow-up clinical data could have limited identifying associations with outcomes and any competing risks between mortality and surgery between the two groups. Moreover, not all included patients had CT data amenable for analysis reducing our sample size significantly. Also, not all ECHO data were amenable for analysis limiting the association we can make between calcium and different $\mathrm{ECHO}$ variables. Echocardiographic data were collected 
at rest, therefore the effect of pericardial calcification on regional RV and LV haemodynamic changes with exercise is not available. In addition, providing a quantitative assessment of calcium severity may have been a more rigorous approach. This is highly appreciated as it is not reasonable to assume that the value of a speckle or two of calcium in a pericardial segment is equivalent to dense large chunks of calcifications in another segment. This is a limitation of our semiquantitative scoring system. Unfortunately, solving this predicament was not possible due to inherent limitations in the calcium scoring software designed initially for coronary calcification and not pericardial calcification. Two examples of these limitations are ${ }^{1}$ : numerous patients had calcifications that were continuous from one pericardial segment to another, especially in the basal LV inferior and basal RV inferior segments, which made splitting the calcifications by the software not feasible; and ${ }^{2}$ the range of values obtained because of the density of the calcifications reached in some cases beyond 30000 Agatston score which extrapolates this system into numerical values not encountered in the coronaries and thus presented a theoretical challenge. Thus, we limited our data analysis to semiquantitative methods of PC scoring, pending development of a more scientifically acceptable method to quantify pericardial calcifications. Future studies addressing the association of distribution of PC with clinical outcomes, myocardial atrophy and ECHO data need to be also further investigated. Also important is the effectiveness of medical therapy in the presence and severity of pericardial calcification.

\section{CONCLUSION}

Our study demonstrates the spatial distribution of pericardial calcification and correlation between calcium and clinical outcomes. Pericardial calcification was found to be located in a circumferential band sparing the basal anterior LV segment and apical regions of the LV and RV. In more than $40 \%$ of patients with pericardial calcifications these calcifications extended into the mitral or tricuspid annuli. Our results showed that the presence of calcium did not significantly predict surgical outcomes and the total PC score did not significantly predict clinical outcomes. A potential protective role of RV calcium in regional myocardial mechanics was observed. Further studies with a quantitative assessment of PC and association with clinical outcomes are needed.

Contributors AS and HAl contributed equally to this study and drafted the manuscript. AS, HAl, AK, AA, CKA and ALK participated in the study design and data collection. DP, PS, DJ and ALK critically reviewed the paper.

Funding The authors have not declared a specific grant for this research from any funding agency in the public, commercial or not-for-profit sectors.

Competing interests None declared.
Patient consent Not required.

Ethics approval Cleveland Clinic Institutional Review Board.

Provenance and peer review Not commissioned; externally peer reviewed.

Data statement The data is secure and confidential and can be only accessed through the Cleveland Clinic pericardial database.

Open access This is an open access article distributed in accordance with the Creative Commons Attribution Non Commercial (CC BY-NC 4.0) license, which permits others to distribute, remix, adapt, build upon this work non-commercially, and license their derivative works on different terms, provided the original work is properly cited, appropriate credit is given, any changes made indicated, and the use is non-commercial. See: http://creativecommons.org/licenses/by-nc/4.0/

\section{REFERENCES}

1. Klein AL, Abbara S, Agler DA, et al. American society of echocardiography clinical recommendations for multimodality cardiovascular imaging of patients with pericardial disease: endorsed by the society for cardiovascular magnetic resonance and society of cardiovascular computed tomography. J Am Soc Echocardiogr. 2013;26:965-1012.

2. Verhaert D, Gabriel RS, Johnston D, et al. The role of multimodality imaging in the management of pericardial disease. Circ Cardiovasc Imaging 2010;3:333-43.

3. Suh SY, Rha SW, Kim JW, et al. The usefulness of three-dimensional multidetector computed tomography to delineate pericardial calcification in constrictive pericarditis. Int J Cardiol 2006;113:414-6.

4. Roberts WC. Pericardial heart disease: its morphologic features and its causes. Proc 2005;18:38-55.

5. Ling LH, Oh JK, Breen JF, et al. Calcific constrictive pericarditis: is it still with us? Ann Intern Med 2000;132:444-50.

6. Chowdhury UK, Subramaniam GK, Kumar AS, et al. Pericardiectomy for constrictive pericarditis: a clinical, echocardiographic, and hemodynamic evaluation of two surgical techniques. Ann Thorac Surg 2006;81:522-9.

7. Szabó G, Schmack B, Bulut C, et al. Constrictive pericarditis: risks, aetiologies and outcomes after total pericardiectomy: 24 years of experience. Eur J Cardiothorac Surg 2013;44:1023-8.

8. Tokuda Y, Miyata H, Motomura N, et al. Outcome of pericardiectomy for constrictive pericarditis in Japan: a nationwide outcome study. Ann Thorac Surg 2013;96:571-6.

9. Bertog SC, Thambidorai SK, Parakh K, et al. Constrictive pericarditis: etiology and cause-specific survival after pericardiectomy. J Am Coll Cardiol 2004;43:1445-52.

10. Ling LH, Oh JK, Schaff HV, et al. Constrictive pericarditis in the modern era: evolving clinical spectrum and impact on outcome after pericardiectomy. Circulation 1999;100:1380-6.

11. Matsuno $\mathrm{Y}$, Shimabukuro $\mathrm{K}$, Ishida N, et al. Off-pump complete pericardiectomy for an unusual case of annular constrictive pericarditis. Ann Thorac Surg 2012;94:e45-e47.

12. Nigri A, Mangieri E, Martuscelli E, et al. Pulmonary trunk stenosis due to constriction by a pericardial band. Am Heart $J$ 1987;114:448-50.

13. Nishimura RA, Kazmier FJ, Smith $\mathrm{HC}$, et al. Right ventricular outflow obstruction caused by constrictive pericardial disease. Am J Cardiol 1985;55:1447-8.

14. Gautam MP, Gautam S, Sogunuru G, et al. Constrictive pericarditis with a calcified pericardial band at the level of left ventricle causing mid-ventricular obstruction. BMJ Case Rep 2012;2012:bcr0920114743.

15. Bogaert J, Meyns B, Dymarkowski S, et al. Calcified constrictive pericarditis: prevalence, distribution patterns, and relationship to the myocardium. JACC Cardiovasc Imaging 2016;9:1013-4.

16. Reuss CS, Wilansky SM, Lester SJ, et al. Using mitral 'annulus reversus' to diagnose constrictive pericarditis. Eur J Echocardiogr 2009;10:372-5.

17. Kusunose K, Dahiya A, Popović ZB, et al. Biventricular mechanics in constrictive pericarditis comparison with restrictive cardiomyopathy and impact of pericardiectomy. Circ Cardiovasc Imaging 2013;6:399-406.

18. Geske JB, Anavekar NS, Nishimura RA, et al. Differentiation of constriction and restriction: complex cardiovascular hemodynamics. J Am Coll Cardiol 2016;68:2329-47. 